International Journal of Pure and Applied Mathematics

Volume 83 No. 2 2013, 331-348

ISSN: 1311-8080 (printed version); ISSN: 1314-3395 (on-line version)

url: http://www.ijpam.eu

doi: http://dx.doi.org/10.12732/ijpam.v83i2.12

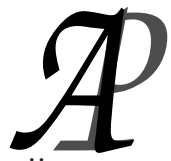

ijpam.eu

\title{
ON THE CONVOLUTION AND INVERSION OF DIAMOND KLEIN GORDON KERNEL
}

\author{
Supaknaree Sattaso ${ }^{1}$, Kamsing Nonlaopon ${ }^{2} \S$ \\ Department of Mathematics \\ Khon Kaen University \\ Khon Kaen 40002, THAILAND
}

Abstract: In this paper, we define the diamond Klein-Gordon Kernel $T_{\alpha}$ and the diamond Klein-Gordon operator of order $\alpha$ on the function $f$ by

$$
D^{\alpha}(f)=T_{\alpha} * f,
$$

where $\alpha \in \mathbb{C}$, the symbol $*$ designates the convolution, and $f \in \mathcal{S}, \mathcal{S}$ is the Schwartz space of functions. In this paper, we aim to study the convolution of $T_{\alpha}$ and obtain the operator $L^{\alpha}=\left[D^{\alpha}\right]^{-1}$ such that if $D^{\alpha}(f)=\varphi$, then $L^{\alpha} \varphi=f$.

AMS Subject Classification: 46F10, 46F 12

Key Words: diamond Klein-Gordon operator, diamond Klein-Gordon kernel, diamond operator, diamond kernel of Marcel Riesz, Dirac delta distribution

\section{Introduction}

The $n$-dimensional ultra-hyperbolic operator $\square^{k}$ iterated $k$ times is defined by

$$
\square^{k}=\left(\frac{\partial^{2}}{\partial x_{1}^{2}}+\frac{\partial^{2}}{\partial x_{2}^{2}}+\cdots+\frac{\partial^{2}}{\partial x_{p}^{2}}-\frac{\partial^{2}}{\partial x_{p+1}^{2}}-\frac{\partial^{2}}{\partial x_{p+2}^{2}}-\cdots-\frac{\partial^{2}}{\partial x_{p+q}^{2}}\right)^{k},
$$

where $p+q=n$ is the dimension of $\mathbb{R}^{n}$ and $k$ is a non-negative integer.

Received: November 8, 2012

(C) 2013 Academic Publications, Ltd. url: www.acadpubl.eu

$\S_{\text {Correspondence author }}$ 
Consider the linear differential equation in the form of

$$
\square^{k} u(x)=f(x)
$$

where $u(x)$ and $f(x)$ are generalized functions and $x=\left(x_{1}, x_{2}, \ldots, x_{n}\right) \in \mathbb{R}^{n}$.

Gel'fand and Shilov [1] were the first to introduce the fundamental solution of (1.2), which is a complicated form. Later, Trione [2] showed that the generalized function $R_{2 k}(x)$, defined by (2.1) with $\alpha=2 k$, is the unique fundamental solution of (1.2) and Tellez [3] also proved that $R_{2 k}(x)$ exists only when $n=p+q$ with odd $p$.

Later, Kananthai [4] was the first to introduce the operator $\diamond^{k}$ called the diamond operator iterated $k$ times, which is defined by

$$
\diamond^{k}=\left[\left(\sum_{i=1}^{p} \frac{\partial^{2}}{\partial x_{i}^{2}}\right)^{2}-\left(\sum_{j=p+1}^{p+q} \frac{\partial^{2}}{\partial x_{j}^{2}}\right)^{2^{k}},\right.
$$

where $n=p+q$ is the dimension of $\mathbb{R}^{n}$, for all $x=\left(x_{1}, x_{2}, \ldots, x_{n}\right)$, and $k$ is a non-negative integer. The operator $\diamond^{k}$ can be expressed in the form

$$
\diamond^{k}=\triangle^{k} \square^{k}=\square^{k} \triangle^{k}
$$

where $\square^{k}$ is defined by (1.1), and

$$
\triangle^{k}=\left(\frac{\partial^{2}}{\partial x_{1}^{2}}+\frac{\partial^{2}}{\partial x_{2}^{2}}+\cdots+\frac{\partial^{2}}{\partial x_{n}^{2}}\right)^{k}
$$

is the Laplace operator iterated $k$ times. On finding the fundamental solution of this product, Kananthai used the convolution of functions which are fundamental solutions of the operators $\square^{k}$ and $\triangle^{k}$. He found that the convolution $(-1)^{k} S_{2 k}(x) * R_{2 k}(x)$ is the fundamental solution of the operator $\diamond^{k}$, that is,

$$
\diamond^{k}\left((-1)^{k} S_{2 k}(x) * R_{2 k}(x)\right)=\delta(x),
$$

where $R_{2 k}(x)$ and $S_{2 k}(x)$ are defined by (2.1) and (2.6), respectively, with $\alpha=2 k$, and $\delta(x)$ is the Dirac delta distribution. The fundamental solution $(-1)^{k} S_{2 k}(x) * R_{2 k}(x)$ is called the diamond kernel of Marcel Riesz. A wealth of effective works on the diamond kernel of Marcel Riesz were presented by Kananthai [5, 6, 7, 8, 9] and Sritanratana and Kananthai [10].

In 1978, Dominguez and Trione [11] introduced the distributional functions $H_{\alpha}(P \pm i 0, n)$, which are causal (anti-causal) analogues of the elliptic kernel of 
Marcel Riesz [12]. Later, Cerutti and Trione [13] defined the causal (anti-causal) generalized Marcel Riesz potentials of order $\alpha, \alpha \in \mathbb{C}$, by

$$
R^{\alpha} \varphi=H_{\alpha}(P \pm i 0, n) * \varphi
$$

where $\varphi \in \mathcal{S}, \mathcal{S}$ is the Schwartz space of functions [14], and $H_{\alpha}(P \pm i 0, n)$ is given by

$$
H_{\alpha}(P \pm i 0, n)=\frac{e^{\mp \alpha \pi i / 2} e^{ \pm q \pi i / 2} \Gamma((n-\alpha) / 2)(P \pm i 0)^{(\alpha-n) / 2}}{2^{\alpha} \pi^{n / 2} \Gamma(\alpha / 2)} .
$$

Here, $P$ is defined by

$$
P=P(x)=x_{1}^{2}+x_{2}^{2}+\cdots+x_{p}^{2}-x_{p+1}^{2}-x_{p+2}^{2}-\cdots-x_{p+q}^{2},
$$

where $q$ is the number of negative terms of the quadratic form $P$. The distributions $(P \pm i 0)^{\lambda}$ are defined by

$$
(P \pm i 0)^{\lambda}=\lim _{\epsilon \rightarrow 0}\left(P \pm i \epsilon|x|^{2}\right)^{\lambda},
$$

where $\epsilon>0, \lambda \in \mathbb{C}$, and $|x|^{2}=x_{1}^{2}+x_{2}^{2}+\cdots+x_{n}^{2}$, see [1]. They also studied the inverse operator of $R^{\alpha}$, denoted by $\left(R^{\alpha}\right)^{-1}$, such that if $f=R^{\alpha} \varphi$, then $\left(R^{\alpha}\right)^{-1} f=\varphi$.

Later, Aguirre [15] defined the ultra-hyperbolic Marcel Riesz operator $M^{\alpha}$ on the function $f$ by

$$
M^{\alpha}(f)=R_{\alpha} * f
$$

where $R_{\alpha}$ is defined by $(2.1)$ and $f \in \mathcal{S}$. He also studied the operator $N^{\alpha}=$ $\left(M^{\alpha}\right)^{-1}$ such that if $M^{\alpha}(f)=\varphi$, then $N^{\alpha} \varphi=f$.

Let us consider the diamond kernel of Marcel Riesz $K_{\alpha, \beta}(x)$ introduced by Kananthai [6], which is given by the convolution

$$
K_{\alpha, \beta}(x)=S_{\alpha}(x) * R_{\beta}(x),
$$

where $S_{\alpha}(x)$ is an elliptic kernel of Marcel Riesz defined by $(2.6)$ and $R_{\beta}(x)$ is the ultra-hyperbolic kernel of Marcel Riesz defined by (2.1). Tellez and Kananthai [16] proved that $K_{\alpha, \beta}(x)$ exists and is in the space of rapidly decreasing distributions. Moreover, they also showed that the convolution of the distributional families $K_{\alpha, \beta}(x)$ relates to the diamond operator.

Later, Maneetus and Nonlaopon [17] defined the diamond Marcel Riesz operator of order $(\alpha, \beta)$ on the function $f$ by

$$
M^{(\alpha, \beta)}(f)=K_{\alpha, \beta} * f,
$$


where $K_{\alpha, \beta}$ is defined by (1.12), $\alpha, \beta \in \mathbb{C}$, and $f \in \mathcal{S}$. They also studied the operator $N^{(\alpha, \beta)}=\left[M^{(\alpha, \beta)}\right]^{-1}$ such that if $M^{(\alpha, \beta)}(f)=\varphi$, then $N^{(\alpha, \beta)} \varphi=f$. Moreover, they defined the Bessel ultra-hyperbolic Marcel Riesz operator of order $\alpha$ on the function $f$ by

$$
U^{\alpha}(f)=R_{\alpha}^{B} * f
$$

where $R_{\alpha}^{B}$ is the Bessel ultra-hyperbolic kernel of Marcel Riesz, $\alpha \in \mathbb{C}$, and $f \in \mathcal{S}$; see [18], for more details. In addition, they studied the operator $E^{\alpha}=$ $\left(U^{\alpha}\right)^{-1}$ such that if $U^{\alpha}(f)=\varphi$, then $E^{\alpha} \varphi=f$. Later, Salao and Nonlaopon [19] defined the Bessel diamond kernel of Marcel Riesz by

$$
K_{\alpha, \beta}^{B}(x)=S_{\alpha}^{B}(x) * R_{\beta}^{B}(x),
$$

where $R_{\beta}^{B}(x)$ and $S_{\alpha}^{B}(x)$ are the Bessel ultra-hyperbolic kernel of Marcel Riesz and the Bessel elliptic kernel of Marcel Riesz, respectively. And they defined the Bessel diamond Marcel Riesz operator of order $(\alpha, \beta)$ on the function $f$ by

$$
U^{(\alpha, \beta)}(f)=K_{\alpha, \beta}^{B} * f,
$$

where $\alpha, \beta \in \mathbb{C}$ and $f \in \mathcal{S}$. In addition, they studied the operator $E^{(\alpha, \beta)}=$ $\left[U^{(\alpha, \beta)}\right]^{-1}$ such that if $U^{(\alpha, \beta)}(f)=\varphi$, then $E^{(\alpha, \beta)} \varphi=f$. Later, Nonlaopon [20] defined the ultra-hyperbolic Klein-Gordon operator of order $\alpha$ on the function $f$ by

$$
T^{\alpha}(f)=W_{\alpha} * f,
$$

where $W_{\alpha}$ is the ultra-hyperbolic Klein-Gordon kernel, $\alpha \in \mathbb{C}$, and $f \in \mathcal{S}$. He also studied the operator $L^{\alpha}(f)=\left[T^{\alpha}\right]^{-1}$ such that if $T^{\alpha}(f)=\varphi$, then $L^{\alpha} \varphi=f$.

In 2007, Tariboon and Kananthai [21] introduced the operator $\left(\diamond+m^{2}\right)^{k}$ called diamond Klein-Gordon operator iterated $k$ times, which is define by

$$
\left(\diamond+m^{2}\right)^{k}=\left[\left(\sum_{i=1}^{p} \frac{\partial^{2}}{\partial x_{i}^{2}}\right)^{2}-\left(\sum_{j=p+1}^{p+q} \frac{\partial^{2}}{\partial x_{j}^{2}}\right)^{2}+m^{2}{ }^{k},\right.
$$

where $p+q=n$ is the dimension of $\mathbb{R}^{n}$, for all $x=\left(x_{1}, x_{2}, \ldots, x_{n}\right) \in \mathbb{R}^{n}, m \geq 0$ and non-negative integers $k$. Later, Nonlaopon et al. [22] studied the fundamental solution of operator $\left(\diamond+m^{2}\right)^{k}$, and this fundamental solution is called the diamond Klein-Gordon kernel. They also studied the Fourier transform of the diamond Klein-Gordon kernel and its convolution [23]. 
Later, Liangprom and Nonlaopon [24] studied properties of the distribution $e^{a x}\left(\diamond+m^{2}\right)^{k} \delta(x)$ by

$$
e^{a x}\left(\diamond+m^{2}\right)^{k} \delta(x)=L^{k} \delta(x)
$$

holds and $e^{a x}\left(\diamond+m^{2}\right)^{k} \delta(x)$ is tempered distribution of oder $4 k$ with support $\{0\}$, where $L$ is the partial differential opertor. And they also showed that boundedness property of the distribution $e^{\alpha x}\left(\diamond+m^{2}\right)^{k} \delta(x)$, where $x=$ $\left(x_{1}, x_{2}, \ldots, x_{n}\right)$ is a variable in $\mathbb{R}^{n}$ and $\alpha=\left(\alpha_{1}, \alpha_{2}, \ldots, \alpha_{n}\right)$ is a constant. In particular, they studied the application of $e^{\alpha x}\left(\diamond+m^{2}\right)^{k} \delta(x)$ for solving the solution of some convolution equation. They found that the type of solution of such convolution equation, such as the ordinary function and the singular distribution, depend on the relationship between $k$ and $M$.

In this paper, we define the diamond Klein-Gordon operator of oder $\alpha$ on the function $f$ by

$$
D^{\alpha}(f)=T_{\alpha} * f
$$

where $T_{\alpha}$ is defined by $(3.1), \alpha \in \mathbb{C}$, and $f \in \mathcal{S}$. Our aim of this paper is to study the convolution of $T_{\alpha}$ and obtain the operator $L^{\alpha}=\left[D^{\alpha}\right]^{-1}$ such that if $D^{\alpha}(f)=\varphi$, then $L^{\alpha} \varphi=f$.

Before we proceed to our main theorem, the following definitions and some concepts require some clarifications.

\section{Preliminaries}

Definition 2.1. Let $x=\left(x_{1}, x_{2}, \ldots, x_{n}\right)$ be a point in the $n$-dimentional Euclidean space $\mathbb{R}^{n}$. Let

$$
u=x_{1}^{2}+x_{2}^{2}+\cdots+x_{p}^{2}-x_{p+1}^{2}-x_{p+2}^{2}-\cdots-x_{p+q}^{2}
$$

be the non-degenerated quadratic form, where $p+q=n$ is the dimension of $\mathbb{R}^{n}$. Let $\Gamma_{+}=\left\{x \in \mathbb{R}^{n}: u>0\right.$ and $\left.x_{i}>0,(i=1,2, \ldots, p)\right\}$ be the interior of forward cone and let $\bar{\Gamma}_{+}$denote its closure. For any complex number $\alpha$, we define

$$
R_{\alpha}(x)= \begin{cases}\frac{u^{(\alpha-\mathrm{n}) / 2}}{K_{\mathrm{n}}(\alpha)}, & \text { for } x \in \Gamma_{+}, \\ 0, & \text { for } x \notin \Gamma_{+},\end{cases}
$$

where

$$
K_{n}(\alpha)=\frac{\pi^{(n-1) / 2} \Gamma((2+\alpha-n) / 2) \Gamma((1-\alpha) / 2) \Gamma(\alpha)}{\Gamma((2+\alpha-p) / 2) \Gamma((p-\alpha) / 2)}
$$


The function $R_{\alpha}(x)$ is called the ultra-hyperbolic kernel of Marcel Riesz and was introduced by Nozaki [25]. It is well known that $R_{\alpha}(x)$ is an ordinary function for $\operatorname{Re}(\alpha) \geq n$ and is a distribution of $\alpha$ for $\operatorname{Re}(\alpha)<n$. Let $\operatorname{supp} R_{\alpha}(x)$ denote the support of $R_{\alpha}(x)$ and suppose that $\operatorname{supp} R_{\alpha}(x) \subset \bar{\Gamma}_{+}$(i.e. $\operatorname{supp} R_{\alpha}(x)$ is compact).

By putting $p=1$ in $R_{\alpha}(x)$ and taking into account Legendre's duplication formula for $\Gamma(z)$, that is,

$$
\Gamma(2 z)=2^{2 z-1} \pi^{-1 / 2} \Gamma(z) \Gamma(z+1 / 2),
$$

we obtain

$$
I_{\alpha}=\frac{v^{(\alpha-n) / 2}}{H_{n}(\alpha)}
$$

and $v=x_{1}^{2}-x_{2}^{2}-x_{3}^{2}-\cdots-x_{n}^{2}$, where

$$
H_{n}(\alpha)=\pi^{(n-2) / 2} 2^{\alpha-1} \Gamma((\alpha+2-n) / 2) \Gamma(\alpha / 2) .
$$

The function $I_{\alpha}$ is called the hyperbolic kernel of Marcel Riesz.

Definition 2.2. Let $x=\left(x_{1}, x_{2}, \ldots, x_{n}\right)$ be a point of $\mathbb{R}^{n}$ and $\omega=$ $x_{1}^{2}+x_{2}^{2}+\cdots+x_{n}^{2}$. The elliptic kernel of Marcel Riesz is defined by

$$
S_{\alpha}(x)=\frac{\omega^{(\alpha-n) / 2}}{W_{n}(\alpha)},
$$

where $n$ is the dimention of $\mathbb{R}^{n}, \alpha \in \mathbb{C}$, and

$$
W_{n}(\alpha)=\frac{\pi^{n / 2} 2^{\alpha} \Gamma(\alpha / 2)}{\Gamma((n-\alpha) / 2)} .
$$

It is well known that $S_{\alpha}(x)$ is an ordinary function for $\operatorname{Re}(\alpha) \geq n$ and is a distribution of $\alpha$ for $\operatorname{Re}(\alpha)<n$.

Note that $n=p+q$. By putting $q=0$ (i.e. $n=p$ ) in (2.1) and (2.2), we can reduce $u^{(\alpha-n) / 2}$ to $\omega^{(\alpha-p) / 2}$, where $\omega=x_{1}^{2}+x_{2}^{2}+\cdots+x_{p}^{2}$, and reduce $K_{n}(\alpha)$ to

$$
K_{p}(\alpha)=\frac{\pi^{(p-1) / 2} \Gamma((1-\alpha) / 2) \Gamma(\alpha)}{\Gamma((p-\alpha) / 2)} .
$$

Using Legendre's duplication formula,

$$
\Gamma(2 z)=2^{2 z-1} \pi^{-1 / 2} \Gamma(z) \Gamma(z+1 / 2)
$$

and

$$
\Gamma(1 / 2+z) \Gamma(1 / 2-z)=\pi \sec (\pi z)
$$


we obtain

$$
K_{p}(\alpha)=\frac{1}{2} \sec (\alpha \pi / 2) W_{p}(\alpha)
$$

Thus, for $q=0$, we have

$$
R_{\alpha}(x)=\frac{u^{(\alpha-p) / 2}}{K_{p}(\alpha)}=2 \cos (\alpha \pi / 2) \frac{u^{(\alpha-p) / 2}}{W_{p}(\alpha)}=2 \cos (\alpha \pi / 2) S_{\alpha}(x) .
$$

In addition, if $\alpha=2 k$ for some non-negative integer $k$, then

$$
R_{2 k}(x)=2(-1)^{k} S_{2 k}(x)
$$

The proof of the Lemmas 2.1 is given in [16].

Lemma 2.1. The convolutions of $K_{\alpha, \beta}(x)$

(i) If $p$ is odd, then

$$
K_{\alpha, \beta}(x) * K_{\alpha^{\prime}, \beta^{\prime}}(x)=\left(R_{\beta+\beta^{\prime}}+A_{\beta, \beta^{\prime}}\right) * S_{\alpha+\alpha^{\prime}},
$$

where $R_{\beta}$ and $S_{\alpha}$ are defined by (2.1) and (2.6), respectively. $A_{\beta, \beta^{\prime}}$ is defined by

$$
A_{\beta, \beta^{\prime}}=-\frac{i}{2} \frac{\sin (\beta \pi / 2) \sin \left(\beta^{\prime} \pi / 2\right)}{\sin \left(\left(\beta+\beta^{\prime}\right) \pi / 2\right)}\left[H_{\beta+\beta^{\prime}}^{+}-H_{\beta+\beta^{\prime}}^{-}\right]
$$

and

$$
H_{\beta}^{ \pm}=H_{\beta}(P \pm i 0, n)
$$

is defined by (1.8).

(ii) If $p$ is even, then

$$
K_{\alpha, \beta}(x) * K_{\alpha^{\prime}, \beta^{\prime}}(x)=B_{\beta, \beta^{\prime}} R_{\beta+\beta^{\prime}} * S_{\alpha+\alpha^{\prime}},
$$

where

$$
B_{\beta, \beta^{\prime}}=\frac{\cos (\beta \pi / 2) \cos \left(\beta^{\prime} \pi / 2\right)}{\cos \left(\left(\beta+\beta^{\prime}\right) \pi / 2\right)} .
$$




\section{The Convolution of $T_{\alpha} * T_{\beta}$}

Let $x=\left(x_{1}, x_{2}, \ldots, x_{n}\right)$ be a point in $\mathbb{R}^{n}$. The function $T_{\alpha}(x, m)$ is defined by

$$
T_{\alpha}(x, m)=\sum_{r=0}^{\infty} m^{2 r}(-1)^{\alpha / 2+r}\left(\begin{array}{c}
-\alpha / 2 \\
r
\end{array}\right) S_{\alpha+2 r}(x) * R_{\alpha+2 r}(x),
$$

where $\alpha$ is a complex parameter and $m$ is a non-negative real number. Here, $R_{\alpha+2 r}(x)$ and $S_{\alpha+2 r}(x)$ is define by (2.1) and (2.6), respectively.

It is well known that $T_{\alpha}(x, m)$ is an ordinary function for $\operatorname{Re}(\alpha) \geq n$ and is a distribution of $\alpha$ for $\operatorname{Re}(\alpha)<n$.

From (3.1) and (1.12), we have

$$
\begin{aligned}
& T_{\alpha}(x, m) * T_{\beta}(x, m) \\
&=\left(\sum_{r=0}^{\infty} m^{2 r}(-1)^{\alpha / 2+r}\left(\begin{array}{c}
-\alpha / 2 \\
r
\end{array}\right) S_{\alpha+2 r}(x) * R_{\alpha+2 r}(x)\right) \\
& *\left(\sum_{s=0}^{\infty} m^{2 s}(-1)^{\beta / 2+s}\left(\begin{array}{c}
-\beta / 2 \\
s
\end{array}\right) S_{\beta+2 s}(x) * R_{\beta+2 s}(x)\right) \\
&=\sum_{r=0}^{\infty} \sum_{s=0}^{\infty} m^{2 r+2 s}(-1)^{(\alpha+\beta) / 2+r+s}\left(\begin{array}{c}
-\alpha / 2 \\
r
\end{array}\right)\left(\begin{array}{c}
-\beta / 2 \\
s
\end{array}\right) \\
&\left\{\left[S_{\alpha+2 r}(x) * R_{\alpha+2 r}(x)\right] *\left[S_{\beta+2 s}(x) * R_{\beta+2 s}(x)\right]\right\} \\
&=\sum_{r=0}^{\infty} \sum_{s=0}^{\infty} m^{2 r+2 s}(-1)^{(\alpha+\beta) / 2+r+s}\left(\begin{array}{c}
-\alpha / 2 \\
r
\end{array}\right)\left(\begin{array}{c}
-\beta / 2 \\
s
\end{array}\right) \\
& {\left[K_{\alpha+2 r, \alpha+2 r}(x) * K_{\beta+2 s, \beta+2 s}(x)\right] . }
\end{aligned}
$$

Here $*$ designates, as usual, the convolution. It is well known that the convolution $K_{\alpha+2 r, \alpha+2 r}(x) * K_{\beta+2 s, \beta+2 s}(x)$ exists for every couple $\alpha, \beta$ of complex number. Therefore, from Lemma 2.1, we have

$$
\begin{aligned}
& K_{\alpha+2 r, \alpha+2 r}(x) * K_{\beta+2 s, \beta+2 s}(x) \\
= & \left(R_{\alpha+\beta+2 r+2 s}(x)-\frac{i}{2} \frac{\sin ((\alpha+2 r) \pi / 2) \sin ((\beta+2 s) \pi / 2)}{\sin ((\alpha+\beta+2 r+2 s) \pi / 2)}\right. \\
& {\left.\left[H_{\alpha+\beta+2 r+2 s}^{+}(x)-H_{\alpha+\beta+2 r+2 s}^{-}(x)\right]\right) * S_{\alpha+\beta+2 r+2 s}(x) }
\end{aligned}
$$

if $p$ is odd, and

$$
K_{\alpha+2 r, \alpha+2 r}(x) * K_{\beta+2 s, \beta+2 s}(x)
$$




$$
\begin{aligned}
= & \frac{\cos ((\alpha+2 r) \pi / 2) \cos ((\beta+2 s) \pi / 2)}{\cos ((\alpha+\beta+2 r+2 s) \pi / 2)} R_{\alpha+\beta+2 r+2 s}(x) \\
& * S_{\alpha+\beta+2 r+2 s}(x)
\end{aligned}
$$

if $p$ is even.

From (3.2) taking into account (3.3) and (3.4), we obtain

$$
\begin{aligned}
& T_{\alpha}(x, m) * T_{\beta}(x, m) \\
= & \sum_{k=0}^{\infty} m^{2 k}(-1)^{(\alpha+\beta) / 2+k}\left[\sum_{r=0}^{k}\left(\begin{array}{c}
-\alpha / 2 \\
r
\end{array}\right)\left(\begin{array}{c}
-\beta / 2 \\
k-r
\end{array}\right)\right]\left[\left(R_{\alpha+\beta+2 k}(x)\right.\right. \\
& \left.\left.-\frac{i}{2} \frac{\sin (\alpha \pi / 2) \sin (\beta \pi / 2)}{\sin ((\alpha+\beta) \pi / 2)}\left[H_{\alpha+\beta+2 k}^{+}(x)-H_{\alpha+\beta+2 k}^{-}(x)\right]\right) * S_{\alpha+\beta+2 k}(x)\right]
\end{aligned}
$$

if $p$ is odd, and

$$
\begin{aligned}
T_{\alpha}(x, m) * T_{\beta}(x, m) & =\frac{\cos (\alpha \pi / 2) \cos (\beta \pi / 2)}{\cos ((\alpha+\beta) \pi / 2)} \sum_{k=0}^{\infty} m^{2 k}(-1)^{(\alpha+\beta) / 2+k} \\
& \times\left[\sum_{r=0}^{k}\left(\begin{array}{c}
-\alpha / 2 \\
r
\end{array}\right)\left(\begin{array}{c}
-\beta / 2 \\
k-r
\end{array}\right)\right] R_{\alpha+\beta+2 k}(x) * S_{\alpha+\beta+2 k}(x)
\end{aligned}
$$

if $p$ is even.

On the other hand,

$$
\sum_{r=0}^{k}\left(\begin{array}{c}
-\alpha / 2 \\
r
\end{array}\right)\left(\begin{array}{c}
-\beta / 2 \\
k-r
\end{array}\right)=\left(\begin{array}{c}
-(\alpha+\beta) / 2 \\
k
\end{array}\right),
$$

from (3.5) and (3.6) and taking into account (3.7), we obtain

$$
\begin{aligned}
& T_{\alpha}(x, m) * T_{\beta}(x, m) \\
= & \sum_{k=0}^{\infty} m^{2 k}(-1)^{(\alpha+\beta) / 2+k}\left(\begin{array}{c}
-(\alpha+\beta) / 2 \\
k
\end{array}\right)\left[\left(R_{\alpha+\beta+2 k}(x)\right.\right. \\
& \left.\left.-\frac{i}{2} \frac{\sin (\alpha \pi / 2) \sin (\beta \pi / 2)}{\sin ((\alpha+\beta) \pi / 2)}\left[H_{\alpha+\beta+2 k}^{+}(x)-H_{\alpha+\beta+2 k}^{-}(x)\right]\right) * S_{\alpha+\beta+2 k}(x)\right] \\
= & {\left[\sum_{k=0}^{\infty} m^{2 k}(-1)^{(\alpha+\beta) / 2+k}\left(\begin{array}{c}
-(\alpha+\beta) / 2 \\
k
\end{array}\right) R_{\alpha+\beta+2 k}(x)\right.}
\end{aligned}
$$




$$
\begin{aligned}
& -\frac{i}{2} \frac{\sin (\alpha \pi / 2) \sin (\beta \pi / 2)}{\sin ((\alpha+\beta) \pi / 2)} \sum_{k=0}^{\infty} m^{2 k}(-1)^{(\alpha+\beta) / 2+k}\left(\begin{array}{c}
-(\alpha+\beta) / 2 \\
k
\end{array}\right) \\
& \left.\left\{H_{\alpha+\beta+2 k}^{+}(x)-H_{\alpha+\beta+2 k}^{-}(x)\right\}\right] * S_{\alpha+\beta+2 k}(x) \\
= & T_{\alpha+\beta}(x, m)-\frac{i}{2} \frac{\sin (\alpha \pi / 2) \sin (\beta \pi / 2)}{\sin ((\alpha+\beta) \pi / 2)} \sum_{k=0}^{\infty} m^{2 k}(-1)^{(\alpha+\beta) / 2+k}\left(\begin{array}{c}
-(\alpha+\beta) / 2 \\
k
\end{array}\right) \\
& {\left[H_{\alpha+\beta+2 k}^{+}(x)-H_{\alpha+\beta+2 k}^{-}(x)\right] * S_{\alpha+\beta+2 k}(x) } \\
= & T_{\alpha+\beta}(x, m)+A_{\alpha, \beta}(x, m),
\end{aligned}
$$

where

$$
\begin{aligned}
A_{\alpha, \beta}(x, m)= & -\frac{i}{2} \frac{\sin (\alpha \pi / 2) \sin (\beta \pi / 2)}{\sin ((\alpha+\beta) \pi / 2)} \sum_{k=0}^{\infty} m^{2 k}(-1)^{(\alpha+\beta) / 2+k}\left(\begin{array}{c}
-(\alpha+\beta) / 2 \\
k
\end{array}\right) \\
& {\left[H_{\alpha+\beta+2 k}^{+}(x)-H_{\alpha+\beta+2 k}^{-}(x)\right] * S_{\alpha+\beta+2 k}(x) }
\end{aligned}
$$

if $p$ is odd, and

$$
\begin{aligned}
& T_{\alpha}(x, m) * T_{\beta}(x, m) \\
& =\frac{\cos (\alpha \pi / 2) \cos (\beta \pi / 2)}{\cos ((\alpha+\beta) \pi / 2)} \sum_{k=0}^{\infty} m^{2 k}(-1)^{(\alpha+\beta) / 2+k}\left(\begin{array}{c}
-(\alpha+\beta) / 2 \\
k
\end{array}\right) R_{\alpha+\beta+2 k}(x) \\
& \quad * S_{\alpha+\beta+2 k}(x) \\
& =\frac{\cos (\alpha \pi / 2) \cos (\beta \pi / 2)}{\cos ((\alpha+\beta) \pi / 2)} T_{\alpha+\beta}(x, m)
\end{aligned}
$$

if $p$ is even.

Putting $\beta=-2 k$ in (3.8) and (3.10), we obtain

$$
T_{\alpha}(x, m) * T_{-2 k}(x, m)=T_{\alpha-2 k}(x, m) .
$$

On the other hand, from [22], we have

$$
T_{-2 k}(x, m)=\left(\diamond+m^{2}\right)^{k} \delta(x), k=1,2, \ldots,
$$

where $\left(\diamond+m^{2}\right)^{k}$ is defined by (1.18).

From (3.11) and (3.12), we have

$$
T_{\alpha}(x, m) *\left(\diamond+m^{2}\right)^{k} \delta(x)=T_{\alpha-2 k}(x, m) .
$$


From (3.13), we have

$$
\left(\diamond+m^{2}\right)^{k} T_{\alpha}(x, m)=T_{\alpha-2 k}(x, m)
$$

In addition, putting $\beta=2 k$ in (3.8) and (3.10), we obtain

$$
T_{\alpha}(x, m) * T_{2 k}(x, m)=T_{\alpha+2 k}(x, m),
$$

and from [22], we have

$$
\left(\diamond+m^{2}\right)^{k} T_{2 k}(x, m)=T_{0}(x, m)=\delta(x) .
$$

From $(3.16), T_{2 k}(x, m)$ is the fundamental solution of the $n$-dimensional diamond-Klein Gordon operator iterated $k$ times.

Furthermore, from [22], we have

$$
T_{\alpha}(x, m=0)=(-1)^{k} S_{2 k}(x) * R_{2 k}(x),
$$

is the fundamental solution of the diamond operator $\diamond^{k}$, where $R_{2 k}(x)$ and $S_{2 k}(x)$ are defined by (2.1) and (2.6), respectively, with $\alpha=2 k$.

\section{The Convolution of $T_{\alpha}(x, m) * T_{\beta}(x, m)$ when $\beta=-\alpha$}

Now, we consider the property of $T_{\alpha}(x, m) * T_{\beta}(x, m)$ when $\beta=-\alpha$.

From (3.8) and (3.10), we know that the following properties are valid:

1. If $p$ is odd and $q$ is even, then

$$
T_{\alpha}(x, m) * T_{\beta}(x, m)=T_{\alpha+\beta}(x, m)+A_{\alpha, \beta}(x, m),
$$

where $A_{\alpha, \beta}(x, m)$ is defined by (3.9).

2. If $p$ and $q$ are both odd, then

$$
T_{\alpha}(x, m) * T_{\beta}(x, m)=T_{\alpha+\beta}(x, m)+A_{\alpha, \beta}(x, m) .
$$

3. If $p$ is even and $q$ is odd, then

$$
T_{\alpha}(x, m) * T_{\beta}(x, m)=\frac{\cos (\alpha \pi / 2) \cos (\beta \pi / 2)}{\cos ((\alpha+\beta) \pi / 2)} T_{\alpha+\beta}(x, m) .
$$


4. If $p$ and $q$ are both even, then

$$
T_{\alpha}(x, m) * T_{\beta}(x, m)=\frac{\cos (\alpha \pi / 2) \cos (\beta \pi / 2)}{\cos ((\alpha+\beta) \pi / 2)} T_{\alpha+\beta}(x, m) .
$$

Moreover, it follows from (3.9) that

$$
\begin{aligned}
& A_{\alpha,-\alpha}(x, m)=\lim _{\beta \rightarrow-\alpha} A_{\alpha, \beta}(x, m) \\
&=-\frac{i}{2} \lim _{\gamma \rightarrow 0} \frac{\sin (\alpha \pi / 2) \sin ((\gamma-\alpha) \pi / 2)}{\sin (\gamma \pi / 2)} \sum_{k=0}^{\infty} m^{2 k}(-1)^{\gamma / 2+k}\left(\begin{array}{c}
-\gamma / 2 \\
k
\end{array}\right) \\
& \quad \times\left[H_{\gamma+2 k}^{+}(x)-H_{\gamma+2 k}^{-}(x)\right] * S_{\gamma+2 k}(x) \\
&=-\frac{i}{2} \lim _{\gamma \rightarrow 0} \frac{\sin (\alpha \pi / 2) \sin ((\gamma-\alpha) \pi / 2)}{\sin (\gamma \pi / 2)} \times \lim _{\gamma \rightarrow 0}\left\{\left[H_{\gamma}^{+}(x)-H_{\gamma}^{-}(x)\right] * S_{\gamma}(x)\right. \\
&+\left.\sum_{k=1}^{\infty} m^{2 k}(-1)^{\gamma / 2+k}\left(\begin{array}{c}
-\gamma / 2 \\
k
\end{array}\right)\left[H_{\gamma+2 k}^{+}(x)-H_{\gamma+2 k}^{-}(x)\right] * S_{\gamma+2 k}(x)\right\} \\
&=-\frac{i}{2} \lim _{\gamma \rightarrow 0} \frac{\sin (\alpha \pi / 2) \sin ((\gamma-\alpha) \pi / 2)}{\sin (\gamma \pi / 2)} \cdot \lim _{\gamma \rightarrow 0}\left\{\left[H_{\gamma}^{+}(x)-H_{\gamma}^{-}(x)\right] * S_{\gamma}(x)\right\},
\end{aligned}
$$

where $\gamma=\alpha+\beta$.

On the other hand, using (2.15) and (1.8), we have

$$
\begin{aligned}
\lim _{\gamma \rightarrow 0}\left[H_{\gamma}^{+}(x)-H_{\gamma}^{-}(x)\right] & =\frac{\Gamma(n / 2)}{\pi^{n / 2}}\left[\lim _{\gamma \rightarrow 0} e^{-\gamma \pi i / 2} e^{q \pi i / 2} \frac{(P+i 0)^{(\gamma-n) / 2}}{\Gamma(\gamma / 2)}\right. \\
& \left.-\lim _{\gamma \rightarrow 0} e^{\gamma \pi i / 2} e^{-q \pi i / 2} \frac{(P-i 0)^{(\gamma-n) / 2}}{\Gamma(\gamma / 2)}\right] \\
& =\frac{\Gamma(n / 2)}{\pi^{n / 2}}\left[\lim _{\gamma \rightarrow 0} e^{-\gamma \pi i / 2} e^{q \pi i / 2} \cdot \frac{\beta=-n / 2}{\operatorname{Res} \Gamma(\beta+n / 2)}\right. \\
& \left.-\lim _{\gamma \rightarrow 0} e^{\gamma \pi i / 2} e^{-q \pi i / 2} \cdot \frac{\operatorname{Res}_{\beta=-n / 2}(P-i 0)^{\beta}}{\operatorname{Res}_{\beta=-n / 2} \Gamma(\beta+n / 2)}\right]
\end{aligned}
$$

Now, taking $n$ as an odd integer, we obtain

$$
\operatorname{Res}_{\lambda=-n / 2-k}(P \pm i 0)^{\lambda}=\frac{e^{ \pm q \pi i / 2} \pi^{n / 2}}{2^{2 k} k ! \Gamma(n / 2+k)} \square^{k} \delta(x),
$$


where $\square^{k}$ is define by (1.1), $p+q=n$, and $k$ is non-negative integer; see [26, 27]. If both $p$ and $q$ are even, then

$$
\operatorname{Res}_{\lambda=-n / 2-k}(P \pm i 0)^{\lambda}=\frac{e^{ \pm q \pi i / 2} \pi^{n / 2}}{2^{2 k} k ! \Gamma(n / 2+k)} \square^{k} \delta(x) .
$$

Nevertheless, If both $p$ and $q$ are odd, then

$$
\operatorname{Res}_{\lambda=-n / 2-k}(P \pm i 0)^{\lambda}=0 .
$$

Therefore, we have

$$
\begin{aligned}
\lim _{\gamma \rightarrow 0}\left[H_{\gamma}^{+}(x)-H_{\gamma}^{-}(x)\right] & =\frac{\Gamma(n / 2)}{\pi^{n / 2}} \cdot \frac{\pi^{n / 2}}{\Gamma(n / 2)}\left[\lim _{\gamma \rightarrow 0} e^{-\gamma \pi i / 2}-\lim _{\gamma \rightarrow 0} e^{\gamma \pi i / 2}\right] \delta(x) \\
& =\lim _{\gamma \rightarrow 0}[-2 i \sin (\gamma \pi / 2)] \delta(x) .
\end{aligned}
$$

From (4.6) and (4.9), we have

$$
\lim _{\gamma \rightarrow 0}\left[H_{\gamma}^{+}(x)-H_{\gamma}^{-}(x)\right]=0
$$

if both $p$ and $q$ are odd ( $n$ even).

By applying (4.10) and (4.11) in (4.5), we have

$$
\begin{aligned}
A_{\alpha,-\alpha}(x, m) & =-\frac{i}{2} \lim _{\gamma \rightarrow 0} \frac{\sin (\alpha \pi / 2) \sin ((\gamma-\alpha) \pi / 2)}{\sin (\gamma \pi / 2)} \\
& \cdot \lim _{\gamma \rightarrow 0}\left\{[-2 i \sin (\gamma \pi / 2)] \delta(x) * S_{\gamma}\right\} \\
& =\sin ^{2}(\alpha \pi / 2) \delta(x) * \delta(x) \\
& =\sin ^{2}(\alpha \pi / 2) \delta(x)
\end{aligned}
$$

if $p$ is odd and $q$ is even, and

$$
A_{\alpha,-\alpha}(x, m)=0
$$

if both $p$ and $q$ are odd.

From (4.1) to (4.4) and using formulae (3.8),(3.10),(3.16),(4.12) and (4.13), if $p$ is odd and $q$ is even, then we obtain

$$
\begin{aligned}
T_{\alpha}(x, m) * T_{-\alpha}(x, m) & =T_{0}(x, m)+A_{\alpha,-\alpha}(x, m) \\
& =\delta(x)+\sin ^{2}(\alpha \pi / 2) \delta(x)
\end{aligned}
$$




$$
=\left(1+\sin ^{2}(\alpha \pi / 2)\right) \delta(x),
$$

if both $p$ and $q$ are odd, then

$$
T_{\alpha}(x, m) * T_{-\alpha}(x, m)=T_{0}(x, m)=\delta(x),
$$

if $p$ is even and $q$ is odd, then

$$
\begin{aligned}
T_{\alpha}(x, m) * T_{-\alpha}(x, m) & =\frac{\cos (\alpha \pi / 2) \cos (-\alpha \pi / 2)}{\cos ((\alpha-\alpha) \pi / 2)} T_{0}(x, m) \\
& =\cos ^{2}(\alpha \pi / 2) \delta(x) .
\end{aligned}
$$

Finally, if both $p$ and $q$ are even, then

$$
\begin{aligned}
T_{\alpha}(x, m) * T_{-\alpha}(x, m) & =\frac{\cos (\alpha \pi / 2) \cos (-\alpha \pi / 2)}{\cos ((\alpha-\alpha) \pi / 2)} T_{0}(x, m) \\
& =\cos ^{2}(\alpha \pi / 2) \delta(x) .
\end{aligned}
$$

\section{Main Results}

Let $D^{\alpha}(f)$ be the diamond Klein-Gordon operator of oder $\alpha$ on the function $f$, which is defined by

$$
D^{\alpha}(f)=T_{\alpha} * f,
$$

where $T_{\alpha}$ is define by (3.1), $\alpha \in \mathbb{C}$, and $f \in S$.

Recall that our objective is to obtain the opertor $L^{\alpha}=\left[D^{\alpha}\right]^{-1}$ such that if $D^{\alpha}(f)=\varphi$, then $L^{\alpha} \varphi=f$ for all $\alpha \in \mathbb{C}$.

We are now ready to state our main theorem.

Theorem 5.1. If $D^{\alpha}(f)=\varphi$ (where $D^{\alpha}(f)$ is defined by (5.1) and $f \in S$ ), then $L^{\alpha} \varphi=f$ such that

$$
L^{\alpha}=\left[D^{\alpha}\right]^{-1}= \begin{cases}{\left[1+\sin ^{2}(\alpha \pi / 2)\right]^{-1} T_{-\alpha},} & \text { if } p \text { is odd and } q \text { is even; } \\ T_{-\alpha}, & \text { if both } p \text { and } q \text { are odd; } \\ \sec ^{2}(\alpha \pi / 2) T_{-\alpha}, & \text { if } p \text { is even with } \alpha \neq 2 s+1\end{cases}
$$

for any non-negative integer $s$. 
Proof. By (5.1), we have

$$
D^{\alpha}(f)=T_{\alpha} * f=\varphi
$$

where $T_{\alpha}$ is defined by (3.1), $\alpha \in \mathbb{C}$, and $f \in S$. If $p$ is odd and $q$ is even, then, in view of (4.14), we obtain

$$
\begin{aligned}
{\left[1+\sin ^{2}(\alpha \pi / 2)\right]^{-1} } & T_{-\alpha} *\left(T_{\alpha} * f\right)=\left[1+\sin ^{2}(\alpha \pi / 2)\right]^{-1}\left(T_{-\alpha} * T_{\alpha}\right) * f \\
& =\left[1+\sin ^{2}(\alpha \pi / 2)\right]^{-1}\left\{\left[1+\sin ^{2}(\alpha \pi / 2)\right] \delta(x)\right\} * f \\
& =\delta(x) * f=f .
\end{aligned}
$$

Hence,

$$
\left[1+\sin ^{2}(\alpha \pi / 2)\right]^{-1} T_{-\alpha}=\left[D^{\alpha}\right]^{-1}=\left(T_{\alpha}\right)^{-1}
$$

for all $\alpha \in \mathbb{C}$.

Similarly, if both $p$ and $q$ are odd, then by (4.15), we obtain

$$
T_{-\alpha} *\left(T_{\alpha} * f\right)=\left(T_{-\alpha} * T_{\alpha}\right) * f=\delta(x) * f=f .
$$

Hence,

$$
T_{-\alpha}=\left[D^{\alpha}\right]^{-1}=\left(T_{\alpha}\right)^{-1}
$$

for all $\alpha \in \mathbb{C}$.

Finally, if $p$ is even, then by (4.16) and (4.17), we have

$$
\begin{aligned}
\sec ^{2}(\alpha \pi / 2) T_{-\alpha} *\left(T_{\alpha} * f\right) & =\sec ^{2}(\alpha \pi / 2)\left(T_{-\alpha} * T_{\alpha}\right) * f \\
& =\sec ^{2}(\alpha \pi / 2)\left\{\cos ^{2}(\alpha \pi / 2) \delta(x)\right\} * f \\
& =\delta(x) * f=f
\end{aligned}
$$

provided that $\alpha \neq 2 s+1$ for any non-negative integer $s$.

Hence,

$$
\sec ^{2}(\alpha \pi / 2) T_{-\alpha}=\left[D^{\alpha}\right]^{-1}=\left(T_{\alpha}\right)^{-1}
$$

for all $\alpha \in \mathbb{C}$ with $\alpha \neq 2 s+1$ for any non-negative integer $s$.

In conclusion, formulae (5.5), (5.7), and (5.9) are the desired results, and this completes the proof. 


\section{References}

[1] I.M. Gel'fand, G.E. Shilov, Generalized Functions, vol. 1, Academic Press, New York, NY, USA (1964).

[2] S.E. Trione, On Marcel Riesz's ultra-hyperbolic kernel, Trab. Math., 116 (1987), 1-12.

[3] M.A. Tellez, The ditributional Hankel transform of Marcel Riesz's ultrahyperbolic kernel, Stud. Appl. Math., 93 (1994), 133-162.

[4] A. Kananthai, On the solution of the $n$-dimensional diamond operator, Appl. Math. Comput., 88, No. 1 (1997), 27-37.

[5] A. Kananthai, On the convolution equation related to the diamond kernel of Marcel Riesz, J. Comput. Appl. Math., 100, No. 1 (1998), 33-39.

[6] A. Kananthai, On the convolution of the diamond kernel of Marcel Riesz, Appl. Math. Comput., 114, No. 1 (2000), 95-101.

[7] A. Kananthai, On the diamond operator related to the wave equation, Nonlinear Anal., 47, No. 2 (2001), 1373-1382.

[8] A. Kananthai, On the Fourier transfrom of the diamond kernel of Marcel Riesz, Appl. Math. Comput., 101, No-s: 2-3 (1999), 151-158.

[9] A. Kananthai, On the green function of the diamond operator related to the Klein-Gordon operator, Bull. Calcutta Math. Soc., 93, No. 5 (2001), 353-360.

[10] G. Sritanratana, A. Kananthai, On the nonlinear diamond operator related to the wave equation, Nonlinear Anal. Real World Appl., 3, No. 4 (2002), 465-470.

[11] A.G. Dominguez, S. E. Trione, On the Laplace transform of retarded invariant functions, Adv. Math., 30, No. 2 (1978), 51-62.

[12] M. Riesz, Integrale de Riemann-Liouville et le probleme de Cauchy, Acta Math., 81 (1949), 1-223.

[13] R.A. Cerutti, S.E. Trione, The inversion of Marcel Riesz ultra-hyperbolic causal operator, Appl. Math. Lett., 12, No. 6 (1999), 25-30.

[14] L. Schwartz, Theories des Distributions, vol. 1, 2, Actualites Scientifiques et Industriel, Hermann \& Cie, Paris, $(1957,1959)$. 
[15] M.A. Aguirre, The inverse ultrahyperbolic Marcel Riesz kernel, Matematiche, $\mathbf{5 4}$ (1999), 55-66.

[16] M.A. Tellez, A. Kananthai, On the convolution product of the distributional families related to the diamond operator, Matematiche, 57 (2002), $39-48$.

[17] D. Maneetus, K. Nonlaopon, The inverse diamond kernel of Marcel Riesz, Int. J. Math. Anal., 7, No. 9 (2013), 441-451.

[18] D. Maneetus, K. Nonlaopon, The inversion of Bessel ultrahyperbolic kernel of Marcel Riesz, Abstr. Appl. Anal., 2011 (2011), Article ID 419157, 13 pages, doi:10.1155/2011/419157.

[19] T. Salao, K. Nonlaopon, On the inverse Bessel diamond kernel of Marcel Riesz, Integral Transforms Spec. Funct., in press.

[20] K. Nonlaopon, On the convolution and inversion of ultra-hyperbolic KleinGordon kernel, Integral Transforms Spec. Funct., Submitted for publication.

[21] J. Tariboon, A. Kananthai, On the Green function of the $\left(\oplus+m^{2}\right)^{k}$ operator, Integral Transforms Spec. Funct., 18, No. 4 (2007), 297-304.

[22] K. Nonlaopon, A. Lunnaree, A. Kananthai, On the solution of the $n$ dimensional diamond Klein-Gordon operator and its convolution, Far East J. Math. Sci., 63, No. 2 (2012), 203-220.

[23] A. Lunnaree, K. Nonlaopon, On the Fourier tranform of the diamond Klein-Gordon kernel, Inter. J. Pure Appl. Math., 68, No. 1 (2011), 8597.

[24] A. Liangprom, K. Nonlaopon, On the convolution equation related to the diamond Klein-Gordon operator, Abstr. Appl. Anal., 2011 (2011), Article ID 908491, 15 pages, doi:10.1155/2011/908491.

[25] Y. Nozaki, On Riemann-Liouville integral of ultra-hyperbolic type, Kodai Mathematical Seminar Reports, 6, No. 2 (1964), 69-87.

[26] M.A. Tellez, The expression and Fourier's transform of $\delta^{(k-1)}\left(m^{2}+P\right)$, Integral Transforms Spec. Funct., 3 (1995), 113-134. 
[27] M.A. Tellez, A.L. Barrenechea, A relation between the $k$ th derivatives of the Dirac delta in $(P \pm i 0)$ and the residue of the distributions $(P \pm i 0)^{\lambda}$, J. Comput. Appl. Math., 108 (1999), 31-40. 